\title{
Disordered Weyl Semimetals and Their Topological Family
}

\author{
Y. X. Zhao ${ }^{*}$ and Z. D. Wang ${ }^{\dagger}$ \\ Department of Physics and Center of Theoretical and Computational Physics, \\ The University of Hong Kong, Pokfulam Road, Hong Kong, China
}

(Received 28 December 2014; published 20 May 2015)

\begin{abstract}
We develop a topological theory for disordered Weyl semimetals in the framework of the gauge invariance of the replica formalism and boundary-bulk correspondence of Chern insulators. An anisotropic topological $\theta$ term is analytically derived for the effective nonlinear $\sigma$ model. It is this nontrivial topological term that ensures that the bulk transverse transport of Weyl semimetals is robust against disorders. Moreover, we establish a general diagram that reveals the intrinsic relations among topological terms in the nonlinear $\sigma$ models and gauge response theories, respectively, for $(2 n+2)$-dimensional topological insulators, $(2 n+1)$-dimensional chiral fermions, $(2 n+1)$-dimensional chiral semimetals, and $(2 n)$-dimensional topological insulators with $n$ being a positive integer.
\end{abstract}

DOI: 10.1103/PhysRevLett.114.206602

PACS numbers: 72.90.+y, 03.65.Vf, 73.43.-f

Introduction.-Recently, Weyl semimetals (WSMs) have been attracting more and more attention due to their interdisciplinary interest from anomalous transport in condensed matter physics [1-10] and quantum field theory anomalies as well as the topological character of their gapless modes [11-20]. The Weyl fermion as the quasiparticle of a WSM has a definite chirality, left handed or right handed, depending on the sign of its spin polarization along the momentum direction [21]. According to the nogo theorem [22], gapless Weyl points in a WSM appear as left- and right-handed pairs in momentum space. Since all of the energy bands of a system with both time-reversal symmetry (TRS) and inversion symmetry (IS) are doubly degenerate for both chiral modes, a WSM can only be realized in a system breaking TRS and/or IS. Here, we focus essentially on the simplest model of a WSM with two Weyl points, whose low-energy effective Hamiltonian reads

$\mathcal{H}_{\mathrm{WSM}}(\mathbf{k}, \mathbf{b})=\left(\begin{array}{ll}-\boldsymbol{\sigma} \cdot(\mathbf{k}-\mathbf{b}(\mathbf{x})) & \\ & \sigma \cdot(\mathbf{k}+\mathbf{b}(\mathbf{x}))\end{array}\right)$,

where the $\sigma$ 's are Pauli matrices and $\mathbf{b}$ is the displacement of Weyl points from the origin of the momentum space. Under the semiclassical approximation, the spatial dependence of $\mathbf{b}(\mathbf{x})$ is assumed to be adiabatic. The model does not have TRS [23]. Each Weyl point has a nontrivial topological charge [13,24], namely, the Chern number on the gapped two-dimensional sphere enclosing a single Weyl point in $\mathbf{k}$ space is \pm 1 , which leads to topological terms in the $U(1)$ response of this model [14-20]. For real materials, disorders are normally unavoidable, and can be studied by the replica method, where a nonlinear $\sigma$ model $(\mathrm{NL} \sigma \mathrm{M})$ encodes fluctuations of Nambu-Goldstone modes that are on a curved manifold [25-27]. Particularly, for topological materials, the global topology of the target manifold may have essential effects, such that topological terms in the $\mathrm{NL} \sigma \mathrm{M}$ are significantly important for describing the physics of the system under disorders [28-31], which highly motivates us to develop a general theory for disordered WSMs.

In this Letter, for the model of Eq. (1) under disorders and preserving no discrete symmetry, we derive for the first time an anisotropic topological term in the action of the $\mathrm{NL} \sigma \mathrm{M}$ as

$$
S_{\mathrm{A \Theta}}=-\frac{1}{8 \pi} \int d^{3} x \epsilon^{i j k} b_{i} \operatorname{tr}\left(Q \partial_{j} Q \partial_{k} Q\right),
$$

where $\epsilon^{i j k}$ is the completely antisymmetric tensor, $b_{i}$ denotes the $i$ th component of $\mathbf{b}$, and $Q$ stands for a $\sigma$ field to be specified later. We refer to this term as an anisotropic $\theta$ term, as it is just a usual $\theta$ term on the plane perpendicular to $\mathbf{b}$ for a constant $\mathbf{b}$ [28]. Actually, this term can be generalized to any chiral semimetal (CSM) with two chiral points separated in a $\mathbf{k}$ space of odd dimensions [32]. We also reveal that this new anisotropic $\theta$ term is associated with the so-called opposite coupling in a CSM, which plays a key role in a relationship diagram of topological terms for a family of topological matter, as illustrated in Fig. 1, where each topological term in the NL $\sigma \mathrm{M}$ has a counterpart in the $\mathrm{U}(1)$ gauge response theory.

Disorder model.-We now consider that the WSM, described by Eq. (1), is subjected to a white-noise and random scalar potential $V(\mathbf{r})$, namely,

$$
\mathcal{H}=\mathcal{H}_{\mathrm{WSM}}(\mathbf{b})+V(\mathbf{r}) .
$$

Assuming that the probability density of $V$ is Gaussian, we have $\overline{V(\mathbf{r})}=0$ and $\overline{V(\mathbf{r}) V\left(\mathbf{r}^{\prime}\right)}=g^{2} \delta^{3}\left(\mathbf{r}-\mathbf{r}^{\prime}\right)$ with $g^{2}$ indicating the strength of the random potential. After applying the replica scenario [25-27] and averaging over the random 


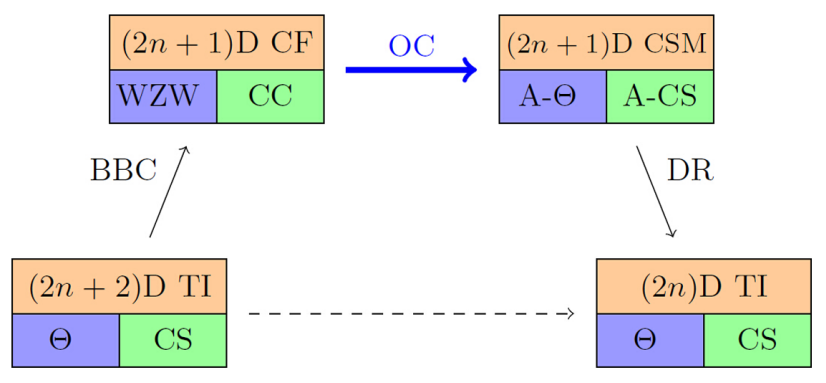

FIG. 1 (color online). Relationship diagram of topological terms and materials for the $n$th unit of a whole topological family of class $A[13,24]$. For each node, the upper, below-left, and below-right boxes denote the model, the topological term in $\mathrm{NL} \sigma \mathrm{M}$, and gauge theory, respectively. The dashed arrow is effectively composed of the three successive solid arrows. Nodes: TI denotes the topological insulator, $\Theta$ represents the theta term, CS the ChernSimons term, CF the chiral fermion, WZW the WZW term, CC the Chern character, CSM the chiral semimetal, $A-\Theta$ the anisotropic $\Theta$, and $A$-CS the anisotropic CS. Arrows: BBC denotes boundarybulk correspondence, OC opposite coupling, and DR dimension reduction. Notably, the thick blue arrow represents a key step that deduces the central result of this work.

potential, the disordered system may be described by a Lagrangian

$$
\mathcal{L}=\psi_{a}^{\dagger} G^{-1} \psi_{a}-\frac{g^{2}}{2} \psi_{a}^{\dagger} \psi_{a} \psi_{b}^{\dagger} \psi_{b},
$$

where the spinor is decomposed into retarded and advanced space, namely, $\psi=\left(\psi^{r}, \psi^{a}\right)^{T}$, and $G^{-1}=\omega-\mathcal{H}_{\mathrm{WSM}}+i \eta \tau_{3}^{r a}$. The Pauli matrix $\tau_{3}^{r a}$ acts in this space, and $\eta$ is an infinitesimal positive number. The subscript as the replica index ranges between 1 and $N$ with the repeated one being summed over. The four-operator term comes from the averaging over the random potential. Since our system is in the symmetry class $A$, we introduce the $\sigma$ field

$$
Q \in \frac{\mathrm{U}(2 N)}{\mathrm{U}(N) \times \mathrm{U}(N)}
$$

to decouple the four-operator term $[28,33,34]$

$$
e^{-\left(g^{2} / 2\right) \int \psi_{\alpha}^{\dagger} \psi_{\beta}^{\dagger} \psi_{\alpha} \psi_{\beta}} \sim \int \mathcal{D} Q e^{-\left(\Delta^{2} / 4 g^{2}\right) \int \operatorname{tr} Q^{2}+\Delta \int \psi_{\alpha}^{\dagger} Q_{\alpha \beta} \psi_{\beta}},
$$

where the Greek subscripts indicate both replica and retarded-advanced indexes. Accordingly, the partition function can be transformed to be in the form

$$
Z=\int \mathcal{D} Q \int \mathcal{D} \psi^{\dagger} \mathcal{D} \psi \exp \left(-\int d^{3} x \mathcal{L}_{f}+\frac{\Delta^{2}}{4 g^{2}} \operatorname{tr} Q Q\right),
$$

where

$$
\mathcal{L}_{f}=\psi_{a}^{\dagger}\left(G^{-1}-\Delta Q_{a b}\right) \psi_{b} .
$$

$\Delta$ is determined by the consistence equation $\pi / g^{2}=$ $\ln \left[1+\left(a_{0} \Delta\right)^{-2}\right]$ with $a_{0}$ being the short-distance cutoff. Assuming that $\omega$ is small and $\Delta>0$, the dynamic terms of the effective $\mathrm{NL} \sigma \mathrm{M}$ are given by

$$
S_{\text {eff }}=\ln \operatorname{Det}\left(\mathcal{H}_{\mathrm{WSM}}+\Delta Q\right) .
$$

It is observed that the operator in the determinant is diagonal in the chirality space, recalling that $\psi_{\alpha}=$ $\left(\xi_{\alpha}, \chi_{\alpha}\right)^{T}$, where $\xi$ and $\chi$ are Weyl spinors with opposite chirality. This means the effective $\mathrm{NL} \sigma \mathrm{M} S_{\text {eff }}$ for $\mathcal{H}_{\mathrm{WSM}}$ is a summation of the NL $\sigma$ Mss for the left- and right-handed chiral components, namely,

$$
S_{\text {eff }}[Q]=S_{-, \text {eff }}[Q,-\mathbf{b}]+S_{+, \text {eff }}[Q, \mathbf{b}],
$$

where the signs in front of $\mathbf{b}$ indicate that it couples oppositely to the left- and right-handed ones. We below derive $S_{ \pm \text {,eff }}$ separately.

Boundary-bulk correspondence.-Before deriving $S_{ \pm, \text {eff }}[Q, \pm \mathbf{b}]$, let us introduce the Wess-Zumino-Witten (WZW) term for a single Weyl point at the center of $\mathbf{k}$ space with disorders but without any antiunitary symmetry, from the viewpoint of boundary-bulk correspondence (BBC) [35]. For a $(2 n+2)$-dimensional topological insulator (TI) in the class $A$ with a nontrivial Chern number $C$ in its bulk [33,34], there are $C$ flavors of chiral fermions with the same chirality on each of its $(2 n+1) \mathrm{D}$ boundaries [36,37]. The $\mathrm{NL} \sigma \mathrm{M}$ of the $(2 n+2) \mathrm{D}$ TI under disorders has a $\theta$ term [28]. In the infrared limit the behavior of the TI is entirely determined by its gapless boundary, and the coupling constant of the $\theta$ term becomes the Chern number $C$ under the renormalization flow [28]. To be concrete, for a 4D TI, the $\theta$ term of the $\mathrm{NL} \sigma \mathrm{M}$ is given by

$$
S_{\Theta}^{4 \mathrm{D}}=\frac{i C}{128 \pi} \int d^{4} x \epsilon^{\mu \nu \rho \lambda} \operatorname{tr} Q \partial_{\mu} Q \partial_{\nu} Q \partial_{\rho} Q \partial_{\lambda} Q .
$$

Viewing from the boundary of the TI, this implies that there exists a WZW term at level $C$ in the NL $\sigma \mathrm{M}$ of the chiral fermions on the boundary [28,29]. For the case of Eq. (6), the corresponding WZW term is found to be

$$
S_{\mathrm{WZW}}=\frac{i \nu_{b}}{128 \pi} \int d \tau d^{3} x \epsilon^{\mu \nu \rho \lambda} \operatorname{tr} \tilde{Q} \partial_{\mu} \tilde{Q} \partial_{\nu} \tilde{Q} \partial_{\rho} \tilde{Q} \partial_{\lambda} \tilde{Q},
$$

where $\tau \in[0,1]$ is the extending parameter, and $Q(x)$ on the $S^{3}$ is extended continuously to $\tilde{Q}(x, \tau)$ with $\tilde{Q}(x, 1)=$ $Q(x)$ and $\tilde{Q}(x, 0)$ being constant [38]. In this case, $\tau$ can be regarded as the parameter of the radial direction if the geometry of the 4D TI is a disk $D^{4}$. Here $\nu_{b}$ is the total $\mathbb{Z}$-type topological charge of the boundary gapless modes, which is defined as the Chern number on the gapped sphere 
enclosing these gapless points in momentum space, and it is equal to the bulk Chern number $C$ according to an established index theorem $[13,24,40]$. Inherently, the $\mathbb{Z}$-type topological charge $\nu_{b}=C$ also implies that the WZW term is at level $\nu_{b}$ in the NL $\sigma \mathrm{M}$. Since this WZW term has a topological character with a nonperturbative discrete coupling constant, gapless chiral modes have a topologically protected finite conductance, free from Anderson localization [29,41]. As the counterpart of the above results for the NL $\sigma \mathrm{M}$, the BBC can also be used to deduce the Chern character (CC) term in the gauge response of $(2 n+1) \mathrm{D}$ chiral fermions from the ChernSimons (CS) term of the gauge response of $(2 n+2) \mathrm{D}$ TIs, since the CS term is not gauge invariant on a manifold with a boundary, leading to the boundary CC terms.

The coupling of disordered Weyl fermions with a gauge field.-If $\mathbf{b}=\mathbf{0}, S_{ \pm, \mathrm{eff}}(Q)$ just corresponds to chiral fermions under disorders. According to the boundary-bulk correspondence, the NL $\sigma \mathrm{M}$ for a model $\mathcal{H}$ with only one gapless point contains a WZW term

$$
\Gamma_{m}[Q]=\frac{i m}{128 \pi} \int d \tau d^{3} x \epsilon^{\mu \nu \rho \lambda} \operatorname{tr} \tilde{Q} \partial_{\mu} \tilde{Q} \partial_{\nu} \tilde{Q} \partial_{\rho} \tilde{Q} \partial_{\lambda} \tilde{Q},
$$

where $m$ is the topological charge of the gapless modes in the model, raising from

$$
S\left[\chi, \chi^{\dagger}\right]=\int d^{3} x \chi^{\dagger}\left(\omega-\mathcal{H}+i \eta \tau_{3}^{r a}-V(\mathbf{r})\right) \chi .
$$

In the present case, $m= \pm$ corresponds to the left- and right-handed fermions, respectively. So it is clear that the WZW terms are canceled if ever $\mathbf{b}=\mathbf{0}$, leading to vanishing topological terms and corresponding to a Dirac Hamiltonian under disorders.

When b is nonzero, we can regard it as a chiral gauge field coupling oppositely to the left- and right-handed fermions. Let us consider $\mathcal{H}_{W,+}=\boldsymbol{\sigma} \cdot \mathbf{k}$ coupled with a $\mathrm{U}(1)$ gauge field $\mathbf{A}$ under disorders, noting that $\mathcal{H}_{W,-}=$ $-\boldsymbol{\sigma} \cdot \mathbf{k}$ can be treated similarly. After application of the replica method, the Lagrangian reads

$$
\mathcal{L}_{+}=\chi_{a}^{\dagger}\left[\omega \delta_{a b}+i \boldsymbol{\sigma} \cdot(\nabla+i \mathbf{A}) \delta_{a b}+i \eta \tau_{3}^{r a}\right] \chi_{b}-\frac{g^{2}}{2} \chi_{a}^{\dagger} \chi_{a} \chi_{b}^{\dagger} \chi_{b},
$$

It is found that the $\mathrm{U}(1)$ gauge transformation can be made for each component of $\chi$ independently; namely, the action is invariant under the transformation

$$
\chi^{a, s} \longrightarrow \chi^{a, s} e^{-i \alpha_{a, s}(\mathbf{x})}
$$

and

$$
\mathbf{A}(\mathbf{x}) \longrightarrow \mathbf{A}(\mathbf{x})-\nabla \alpha_{a, s},
$$

with the superscript " $s$ " being the index of the retardedadvanced space. Accordingly in the nonlinear $\sigma$ version,
$Q^{(a, s)\left(a^{\prime} s^{\prime}\right)} \sim \psi^{\dagger a, s} \psi^{a^{\prime}, s^{\prime}}$ transforms under the gauge transformation as

$$
Q \longrightarrow e^{i \alpha_{a, s}} Q^{(a, s)\left(a^{\prime} s^{\prime}\right)} e^{-i \alpha_{a^{\prime}, s^{\prime}}}
$$

In the absence of the gauge field, the $\mathrm{NL} \sigma \mathrm{M}$ is

$$
S_{+, \mathrm{eff}}[Q]=\frac{1}{\lambda_{+}} \int d^{3} x \operatorname{tr}\left(\partial_{j} Q \partial_{j} Q\right)+\Gamma_{+}[Q],
$$

where $1 / \lambda_{+}$determined by the microscopic details of the Weyl point is proportional to the longitudinal conductivity. Our strategy is to find the minimal coupling of the NL $\sigma \mathrm{M}$ with the $U(1)$ gauge field, which is invariant under the above gauge transformation. To simplify our calculation but without loss of generality, we consider a specific gauge transformation as

$$
\mathbf{A}(\mathbf{x}) \longrightarrow \mathbf{A}(\mathbf{x})-\nabla \alpha \tau_{3}^{r a}
$$

and

$$
Q \longrightarrow e^{i \tau_{3}^{a r} \alpha} Q e^{-i \tau_{3}^{a r} \alpha},
$$

which are the opposite constants in advanced and retarded spaces, respectively. Following a classic work on current algebra of Witten [35], we highlight our derivations below. The infinitesimal variation of $Q$ is

$$
Q \longrightarrow Q+i \alpha\left[\tau_{3}, Q\right]
$$

$\tau_{3}$ operates on retarded and advanced spaces, and hereafter we drop the superscript for simplicity. Since the minimal coupling for the ordinary term is readily obtained by the substitution $\partial_{j} \longrightarrow \hat{\partial}_{j}=\partial_{j}+\left[i A_{j}\right.$, ], we thus focus only on the WZW term. The variation of $\Gamma_{+}$under the infinitesimal local gauge transformation is

$$
\delta \Gamma_{+}=-\frac{1}{16 \pi} \int_{S^{3}} \operatorname{tr}\left(\mathbf{d} \alpha \tau_{3 \wedge} Q_{\wedge} \mathbf{d} Q_{\wedge} \mathbf{d} Q\right),
$$

where the exterior derivative and wedge product have been used for brevity. Thus, we have

$$
J_{\mathrm{WZW}}=-\frac{1}{16 \pi} Q \mathbf{d} Q_{\wedge} \mathbf{d} Q .
$$

We may expect that the coupling takes the form $\int \operatorname{tr}\left(\mathbf{A}_{\wedge} J\right)$. However, since the current $J_{\mathrm{WZW}}$ is not gauge invariant, additional terms are needed to cancel its variation under the gauge transformation, which turns out to be $(1 / 4 \pi) \int \operatorname{tr}\left(\mathbf{A}_{\wedge} \mathbf{d} \mathbf{A} Q\right)$. As a result, the total gauge invariant action for the \pm case is given by 


$$
\begin{aligned}
S_{ \pm, \mathrm{eff}}= & \frac{1}{\lambda_{ \pm}} \int d^{3} x \operatorname{tr}\left(\hat{\partial}_{j} Q \hat{\partial}_{j} Q\right) \pm \frac{1}{4 \pi} \int \operatorname{tr}\left(\mathbf{A}_{\wedge} \mathbf{d} \mathbf{A} Q\right) \\
& \mp \frac{1}{16 \pi} \int \operatorname{tr}\left(\mathbf{A}_{\wedge} Q \mathbf{d} Q_{\wedge} \mathbf{d} Q\right)+\Gamma_{ \pm} .
\end{aligned}
$$

Opposite coupling.-We are now ready to obtain the $\mathrm{NL} \sigma \mathrm{M}$ of the WSM. In the WSM $\mathcal{H}_{+}\left(\mathcal{H}_{-}\right)$is coupled with $+\mathbf{b}(-\mathbf{b})$. Thus, from Eqs. (5) and (8), it is found that

$$
S_{\mathrm{eff}}[Q]=\frac{1}{\lambda} \int d^{3} x \operatorname{tr}\left(\partial_{j} Q \partial_{j} Q\right)+S_{\mathrm{A \Theta}}[Q],
$$

where $1 / \lambda=1 / \lambda_{+}+1 / \lambda_{-}$, recalling that $S_{\mathrm{A} \Theta}$, as our most main result, is given by Eq. (2). Also, note that the first term in Eq. (9) is actually a normal action that accounts for usual nontopological properties.

The physical meaning of the anisotropic $\theta$ term becomes clear if $\mathbf{b}$ is constant. Intuitively, a $2 \mathrm{D}$ slice at any $\mathbf{k} \in$ $(-\mathbf{b}, \mathbf{b})$ along the $\mathbf{b}$ direction may be viewed as a $2 \mathrm{D} \mathrm{TI}$ with unit Chern number, which is accompanied by a bulk transverse conductivity and edge chiral gapless modes that form a Fermi arc on the surface [see the latter discussion around Eq. (11)]. It is interesting to note that the transverse conductivity in the plane perpendicular to $\mathbf{b}$ is proportional to the magnitude of $\mathbf{b}$, being completely independent of the disorder strength $g$. The anisotropic form in the bulk is also consistent with the edge currents traveling perpendicular to b, enabling them to have topological protection similar to that of the WZW term. On the other hand, it is seen from the derivation that the WZW terms of the two Weyl points are canceled, which relies only on the fact that the two Weyl points have opposite topological charges, independent of their positions in $\mathbf{k}$ space. When the WZW term is absent after the opposite coupling, its corresponding topological protection is lost, consistent with the fact that disorders may mix the two Weyl points leading to localization [42]. However, remarkably, the new nontrivial anisotropic $\theta$ term emerges, such that it embodies the remaining anisotropic topological protection originating from the topological charges after the global cancellation, analogous to the net electric field generated from an electric dipole.

At this stage, we elaborate on how to derive the A-CS term in Fig. 1 (see the Supplemental Material [43] for details), from the trick of opposite coupling. Treating $\mathbf{b}$ as a gauge field coupling oppositely to the two Weyl points, the corresponding Lagrangian reads $\mathcal{L}=\bar{\psi}\left(i \not \partial-\not \partial-\gamma^{5} b\right) \psi$, where $\mathbf{b}$ is promoted to be a space-time vector $b_{\mu}$ with $b_{0}$ corresponding to the deviation in energy, and the notations for the Dirac matrices are consistent with those in Ref. [21]. There are two types of vertices, The square vertex represents $-\int d^{4} x \bar{\psi} \gamma^{5} b y$ and the circle vertex represents $-\int d^{4} x \bar{\psi} A \psi$. The $A$-CS term is given by the three-vertex diagrams with two circle vertices and one square vertex, shown in Fig. 2. As the $\mathrm{U}(1)$ gauge symmetry is

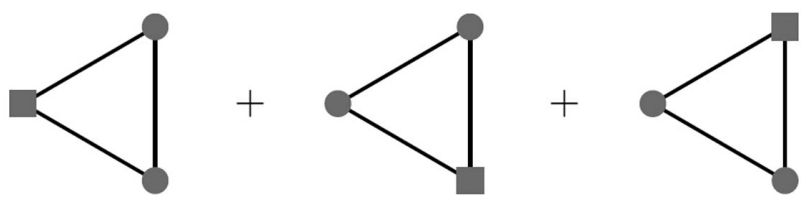

FIG. 2. Three-vertex diagrams.

fundamental, we adopt the dimensional regularization scheme for the internal momentum $l$ in the loop. Accordingly, $l$ is decomposed as $l=l_{\|}+l_{\perp}$. In the above notations, $l_{\|}$is still in the four-dimensional spacetime, but $l_{\perp}$ is in the extended infinitesimal dimensions, anticommuting with $\gamma^{\mu}$ and commuting with $\gamma^{5}[21,44]$. The three diagrams contribute equally, and after a dramatic cancellation only a finite term is left, similar to the derivation of the axial anomaly $[11,12,21]$, leading to the anisotropic CS term

$$
S_{\mathrm{ACS}}=-\frac{1}{4 \pi^{2}} \int d^{4} x \epsilon^{\mu \nu \rho \sigma} b_{\mu} A_{\nu} \partial_{\rho} A_{\sigma}(x) .
$$

Dimension reduction.-The last arrow process to be established is the dimension reduction from a $(2 n+1) \mathrm{D}$ CSM to a $(2 n) \mathrm{D}$ TI in class $A$. First, it is observed that if $\mathbf{b}$ is a constant vector in the model (1), along the direction of $\mathbf{b}$, we may regard the 3D system as a collection of 2D systems perpendicular to $\mathbf{b}$ in $\mathbf{k}$ space, and thus the 2D systems gapped over $\mathbf{k} \in(-\mathbf{b}, \mathbf{b})$ are $2 \mathrm{D}$ TIs with unit Chern number and the others outside this range are trivial, except at the two gapless points. Setting $\mathbf{b}=(0,0, b)$ constant and all fields independent of $z$ in Eqs. (2) and (10), the dimension reduction gives $S_{\mathrm{A} \Theta}=-\left(b L_{z} / 8 \pi\right) \int d^{2} x \epsilon^{j k} \operatorname{tr}\left(Q \partial_{j} Q \partial_{k} Q\right)$ and $S_{\mathrm{ACS}}=-\left(b L_{z} / 4 \pi^{2}\right) \int d^{3} x \epsilon^{\nu \rho \sigma} A_{\nu} \partial_{\rho} A_{\sigma}$. Since $k_{z} \in$ $(-b, b)$ correspond to a collection of unit topological insulators (Chern insulators), divided by the dimension constant $2 b \times\left(L_{z} / 2 \pi\right)$, the above equations give the well-known $\theta$ term and CS term for 2D TIs

$$
\begin{aligned}
& S_{\Theta}^{2 \mathrm{D}}=-\frac{1}{8} \int d^{2} x \epsilon^{j k} \operatorname{tr}\left(Q \partial_{j} Q \partial_{k} Q\right), \\
& S_{\mathrm{CS}}^{2 \mathrm{D}}=-\frac{1}{4 \pi} \int d^{3} x \epsilon^{\mu \nu \rho} A_{\mu} \partial_{\nu} A_{\rho}(x) .
\end{aligned}
$$

Since the above treatment is applicable for any integer $n>0$, the dimension reduction (DR) from a $(2 n+1) \mathrm{D}$ CSM to a $(2 n) \mathrm{D}$ TI in Fig. 1 has been completed. The dimension reduction actually illustrates the correspondence between the anisotropic $\theta$ term of Eq. (2) and the anisotropic CS term of Eq. (10), recalling that the CS term for the 2D TI indicates the transverse conductivity and the $\theta$ term implies the stability of this transverse transportation under disorders.

Remark.-We now comment on the essence of the emergent topological terms. In quantum field theory, this emergence is usually related to some quantum anomaly, 
where two regularization schemes with different symmetries are contradictory $[11,12,28]$. From the viewpoint of band theory, these contradictions usually originate from nontrivial topological configurations of the Berry fiber bundle. The coupling constant of a topological term can formally be expressed as a topological invariant of the Berry fiber bundle, which affects directly the transport properties [45], and thus its nontrivial topological configurations may account for anomalous transport in quantum anomalies.

This work was supported by the GRF (Grants No. HKU7045/13P and No. HKU173051/14P) and the CRF (Grant No. HKU8/11G) of Hong Kong.

*yuxinphy@hku.hk tzwang@hku.hk

[1] S. Murakami, New J. Phys. 9, 356 (2007).

[2] X. Wan, A. M. Turner, A. Vishwanath, and S. Y. Savrasov, Phys. Rev. B 83, 205101 (2011).

[3] W. W. Krempa and Y.-B. Kim, Phys. Rev. B 85, 045124 (2012).

[4] A. A. Burkov and L. Balents, Phys. Rev. Lett. 107, 127205 (2011).

[5] A. A. Burkov, M. D. Hook, and L. Balents, Phys. Rev. B 84, 235126 (2011).

[6] A. A. Zyuzin, S. Wu, and A. A. Burkov, Phys. Rev. B 85, 165110 (2012).

[7] G. B. Halasz and L. Balents, Phys. Rev. B 85, 035103 (2012).

[8] F. Haldane, arXiv:1401.0529.

[9] S. Y. Xu et al., arXiv:1502.03807.

[10] C. Zhang et al., arXiv:1503.02630.

[11] S. Adler, Phys. Rev. 177, 2426 (1969).

[12] J. S. Bell and R. Jackiw, Nuovo Cimento A 60, 4 (1969).

[13] G. E. Volovik, The Universe in a Helium Droplet (Clarendon, Oxford, 2003).

[14] S. M. Carroll, G. B. Field, and R. Jackiw, Phys. Rev. D 41, 1231 (1990).

[15] R. Jackiw and S.-Y. Pi, Phys. Rev. D 68, 104012 (2003).

[16] C.-X. Liu, P. Ye, and X.-L. Qi, Phys. Rev. B 87, 235306 (2013).

[17] A. A. Zyuzin and A. A. Burkov, Phys. Rev. B 86, 115133 (2012).

[18] D. T. Son and N. Yamamoto, Phys. Rev. Lett. 109, 181602 (2012).

[19] A. G. Grushin, Phys. Rev. D 86, 045001 (2012).

[20] M. M. Vazifeh and M. Franz, Phys. Rev. Lett. 111, 027201 (2013).
[21] M.E. Peskin and D. V. Schroeder, An Introduction to Quantum Field Theory (Westview Press, Boulder, Colorado, USA, 1995).

[22] H. B. Nielsen and M. Ninomiya, Phys. Lett. B 105, 219 (1981).

[23] Although the displacements of two Weyl points are assumed to be placed oppositely in momentum space due to IS for simplicity, our main results are valid for the general case without IS.

[24] Y. X. Zhao and Z. D. Wang, Phys. Rev. Lett. 110, 240404 (2013).

[25] A. M. M. Pruisken and L. Schafer, Phys. Rev. Lett. 46, 490 (1981).

[26] A. M. M. Pruisken and L. Schafer, Nucl. Phys. B200, 20 (1982).

[27] See F. Wegner, Z. Phys. B 35, 207 (1979); 36, 209 (1980) and references therein.

[28] A. M. M. Pruisken, Nucl. Phys. B235, 277 (1984).

[29] A. P. Schnyder, S. Ryu, A. Furusaki, and A. W. W. Ludwig, Phys. Rev. B 78, 195125 (2008); AIP Conf. Proc. 1134, 10 (2009); S. Ryu, A. P. Schnyder, A. Furusaki, and A. W. W Ludwig, New J. Phys. 12, 065010 (2010).

[30] S. Ryu, C. Mudry, H. Obuse, and A. Furusaki, Phys. Rev. Lett. 99, 116601 (2007).

[31] P. M. Ostrovsky, I. V. Gornyi, and A. D. Mirlin, Phys. Rev. Lett. 98, 256801 (2007).

[32] Here the term "chiral point" refers to a gapless point in any odd dimensional momentum space such that the topological charge is nontrivial.

[33] M. R. Zirnbauer, J. Math. Phys. (N.Y.) 37, 4986 (1996).

[34] A. Altland and M. R. Zirnbauer, Phys. Rev. B 55, 1142 (1997).

[35] E. Witten, Nucl. Phys. B223, 422 (1983).

[36] M. Z. Hasan and C. L. Kane, Rev. Mod. Phys. 82, 3045 (2010).

[37] X. L. Qi and S. C. Zhang, Rev. Mod. Phys. 83, 1057 (2011).

[38] For a direct mathematical derivation of Eq. (7), see Ref. [39].

[39] Y. X. Zhao and Z. D. Wang, arXiv:1504.02373.

[40] Y.X. Zhao and Z.D. Wang, Phys. Rev. B 89, 075111 (2014); 90, 115158 (2014).

[41] E. Witten, Commun. Math. Phys. 92, 455 (1984).

[42] Z. Huang, D. P. Arovas, and A. V. Balatsky, New J. Phys. 15, 123019 (2013).

[43] See Supplemental Material at http://link.aps.org/ supplemental/10.1103/PhysRevLett.114.206602for the derivation details of the anisotropic Chern-Simons term using the opposite coupling.

[44] G. 't Hooft and M. J. G. Veltman, Nucl. Phys. B44, 198 (1972).

[45] D. Xiao, M.-C. Chang, and Q. Niu, Rev. Mod. Phys. 82, 1959 (2010). 